Ferrata Storti Foundation

\title{
Hemodynamic provocation with acetazolamide shows impaired cerebrovascular reserve in adults with sickle cell disease
}

Haematologica 2019

Volume 104(4):690-699

\section{Correspondence:}

LENA VACLAVU

I.vaclavu@amc.uva.nl

Received: September 5, 2018.

Accepted: November 23, 2018.

Pre-published: December 6, 2018.

doi:10.3324/haematol.2018.206094

Check the online version for the most updated information on this article, online supplements, and information on authorship \& disclosures: www. haematologica.org/content/104/4/690

(C)2019 Ferrata Storti Foundation

Material published in Haematologica is covered by copyright. All rights are reserved to the Ferrata Storti Foundation. Use of published material is allowed under the following terms and conditions:

https://creativecommons.org/licenses/by-nc/4.0/legalcode. Copies of published material are allowed for personal or internal use. Sharing published material for non-commercial purposes is subject to the following conditions:

https://creativecommons.org/licenses/by-nc/4.0/leǵalcode, sect. 3. Reproducing and sharing published material for commercial purposes is not allowed without permission in writing from the publisher.

\author{
Lena Václavi̊, ${ }^{1}$ Benoit N. Meynart, ${ }^{1}$ Henri J.M.M. Mutsaerts, ${ }^{1}$ \\ Esben Thade Petersen, ${ }^{2}$ Charles B.L.M. Majoie, ${ }^{1}$ Ed T. VanBavel, ${ }^{3}$ \\ John C. Wood, ${ }^{4}$ Aart J. Nederveen ${ }^{1}$ and Bart J. Biemond ${ }^{5}$
}

${ }^{1}$ Amsterdam UMC, Radiology and Nuclear Medicine, University of Amsterdam, the Netherlands; ${ }^{2}$ Danish Research Centre for Magnetic Resonance, Centre for Functional and Diagnostic Imaging and Research, Copenhagen University Hospital Hvidovre, Denmark; ${ }^{3}$ Amsterdam UMC, Biomedical Engineering and Physics, University of Amsterdam, the Netherlands; ${ }^{4}$ Cardiology and Radiology, Children's Hospital of Los Angeles, CA, USA; ${ }^{5}$ Amsterdam UMC, Hematology, Internal Medicine, University of Amsterdam, the Netherlands

\section{ABSTRACT}

ickle cell disease is characterized by chronic hemolytic anemia and vascular inflammation, which can diminish the vasodilatory capac$\checkmark$ ity of the small resistance arteries, making them less adept at regulating cerebral blood flow. Autoregulation maintains adequate oxygen delivery, but when vasodilation is maximized, the low arterial oxygen content can lead to ischemia and silent cerebral infarcts. We used magnetic resonance imaging of cerebral blood flow to quantify whole-brain cerebrovascular reserve in 36 adult patients with sickle cell disease (mean age, $31.9 \pm 11.3$ years) and 11 healthy controls (mean age, $37.4 \pm 15.4$ years), and we used high-resolution 3D FLAIR magnetic resonance imaging to determine the prevalence of silent cerebral infarcts. Cerebrovascular reserve was calculated as the percentage change in cerebral blood flow after a hemodynamic challenge with acetazolamide. Coregistered lesion maps were used to demonstrate prevalent locations for silent cerebral infarcts. Cerebral blood flow was elevated in patients with sickle cell disease compared to controls (median [interquartile range]: 82.8 [20.1] vs. 51.3 [4.8] mL/100g/min, $P<0.001)$. Cerebral blood flow was inversely associated with age, hemoglobin, and fetal hemoglobin, and correlated positively with bilirubin, and $\mathrm{LDH}$, indicating that cerebral blood flow may reflect surrogates of hemolytic rate. Cerebrovascular reserve in sickle cell disease was decreased by half compared to controls (34.1 [33.4] vs. 69.5 [32.4] \%, P<0.001) and was associated with hemoglobin and erythrocyte count indicating anemia-induced hemodynamic adaptations. In total, 29/36 patients (81\%) and 5/11 controls $(45 \%)$ had silent cerebral infarcts (median volume of 0.34 vs. 0.02 $\mathrm{mL}, P=0.03$ ). Lesions were preferentially located in the borderzone. In conclusion, patients with sickle cell disease have a globally reduced cerebrovascular reserve as determined by arterial spin labeling with acetazolamide and reflects anemia-induced impaired vascular function in sickle cell disease. This study was registered at clinicaltrials.gov identifier 02824406

\section{Introduction}

Sickle Cell Disease (SCD) is associated with chronic hemolytic anemia and vascular inflammation, ${ }^{1}$ with progressive multiorgan damage including nephropathy, pulmonary hypertension, priapism, leg ulcers, and stroke. ${ }^{2}$ Manifestations of progressive cerebral injury in SCD include overt stroke as well as silent cerebral 
Table 1. Patient characteristics and baseline measurements.

\begin{tabular}{|c|c|c|c|}
\hline & Controls & Patients with SCD & $P^{*}$ \\
\hline Characteristic & $\mathrm{n}=11$ & $\mathrm{n}=36$ & \\
\hline Age, years & $37.4 \pm 15.4$ & $31.9 \pm 11.3$ & 0.52 \\
\hline Sex & 6 men, 5 women & 23 men, 12 women & $0.46^{++}$ \\
\hline Bodyweight, kg & $76(14)$ & $70(18)$ & $<0.01$ \\
\hline \multicolumn{4}{|l|}{ Ethnicity, n (\%) } \\
\hline South America: Suriname & $7(64)$ & $19(54)$ & 0.59 \\
\hline Western Africa: Benin, Congo, Ghana, Guinea, Nigeria, Sierra Leone & $2(18)$ & $11(31)$ & 0.39 \\
\hline Caribbean: Dutch Antilles, Jamaica & $1(9)$ & $4(11)$ & 0.83 \\
\hline Europe: Turkey & $1(9)$ & $1(3)$ & 0.38 \\
\hline \multicolumn{4}{|l|}{ Medication \& therapy } \\
\hline Hydroxyurea, n (\%) & - & $13(37)$ & - \\
\hline Chronic blood transfusion therapy, n (\%) & - & $3(9)$ & - \\
\hline \multicolumn{4}{|l|}{ Cardiovascular risk factors } \\
\hline Systolic blood pressure, mmHg & $133 \pm 10$ & $121 \pm 10$ & $<0.01$ \\
\hline Diastolic blood pressure, mmHg & $87 \pm 7$ & $71 \pm 8$ & $<0.001$ \\
\hline MAP, mmHg & $102 \pm 7$ & $87 \pm 7$ & $<0.001$ \\
\hline Heart rate, bpm & $72 \pm 17$ & $76 \pm 11$ & 0.51 \\
\hline Nicotine smokers, n (\%) & $1(9)$ & $9(26)$ & $0.26^{++}$ \\
\hline Cannabis smokers, n (\%) & $1(9)$ & $4(11)$ & $0.85^{++}$ \\
\hline \multicolumn{4}{|l|}{ Hematologic characteristics } \\
\hline \multirow[t]{2}{*}{ Genotype } & $\operatorname{HbAA}(\mathrm{n}=9,82 \%)$ & $\operatorname{HbSS}(\mathrm{n}=31,89 \%)$ & - \\
\hline & $\operatorname{HbAS}(\mathrm{n}=2,18 \%)$ & $\operatorname{HbS} 0(\mathrm{n}=4,11 \%)$ & - \\
\hline Hemoglobin, g/dL & $13.6 \pm 1.3$ & $8.8 \pm 1.4 \downarrow$ & $<0.001$ \\
\hline Reticulocyte count, \% & $1.3 \pm 0.5$ & $8.9 \pm 4.2 \uparrow$ & $<0.001$ \\
\hline Reticulocyte count, \#10\%/L & $60 \pm 25$ & $261 \pm 108 \uparrow$ & $<0.001$ \\
\hline Bilirubin total, mg/dL & $0.6 \pm 0.5$ & $3.1 \pm 2.0 \uparrow$ & $<0.001$ \\
\hline ASAT, U/L & $39.3 \pm 31.1$ & $48.1 \pm 16.2 \uparrow$ & $<0.05$ \\
\hline LDH & $190 \pm 31$ & $459 \pm 165 \uparrow$ & $<0.05$ \\
\hline
\end{tabular}

${ }^{++} \chi^{2}$ test. ${ }^{*}$ T-test, or Wilcoxon signed rank test was used to test the statistical significance of the difference as appropriate. MAP: mean arterial pressure: [(2 $*$ DiastolicBP) + SystolicBP) ]/3 mmHg. $\uparrow$ above healthy reference / $\downarrow$ below healthy reference. LDH: Lactate dehydrogenase.

infarcts (SCI). ${ }^{3,4}$ Although SCI were once thought to be benign, SCI volume is associated with reduced cognitive performance in children with $\mathrm{SCD}^{5}$ and a 14 -fold increased risk of overt stroke in pediatric SCD. ${ }^{6}$ SCI risk increases relentlessly with age, ${ }^{7,8}$ reaching a prevalence of $50 \%$ by the age of $30 .{ }^{9}$ There is currently no treatment for SCIs in adults, although efforts to reduce their incidence by blood transfusions have been made. ${ }^{10}$ Nevertheless, identifying modifiable risk factors and predictors of these lesions is a focus of current research in adult SCD.

Cerebrovascular reserve (CVR) is a measure of the viability of cerebral vessels to respond to a vasoactive stimulus and is often used to study hemodynamic status in neurovascular diseases. ${ }^{11} \mathrm{CVR}$ is defined as the remaining vasodilating capacity of the cerebral arterioles in response to an exogenous stimulus such as $\mathrm{CO}_{2}$ or acetazolamide. ${ }^{12}$ Recent studies suggest that impaired CVR predicts locations of lesions at one year follow up ${ }^{13}$ and the risk of stroke in steno-occlusive disease, ${ }^{14,15}$ providing compelling evidence that this hemodynamic marker can inform future cerebral damage observed on MRI.

However, the predictive value of CVR in patients with SCD has not yet been shown. Previous cross-sectional studies have observed reduced CVR in children and adults with SCD without a history of overt stroke, ${ }^{16-20}$ and we have recently shown dilated cerebral vessels at baseline in SCD. ${ }^{21}$ Together, these findings suggest that high resting blood flow demands are met by vasodilation. Vasodilation at rest will limit further dilation in times of increased demand, which poses a risk for ischemia. Examples of such risk in SCD include infection, fever, acute anemic events, ${ }^{22}$ and obstructive sleep apnea. ${ }^{23}$ CVR was previously associated with anemia, ${ }^{20}$ and dilatory function of the vessels could additionally be impaired in SCD due to vascular inflammation, low nitric oxide and abnormal endothelial function. Since the brain cannot store its own oxygen, it must maintain constant perfusion, and inadequate oxygen delivery by increases in demand or low hemoglobin may cause ischemia and SCIs due to a lack of vasodilatory reserve. We hypothesized that CVR is lower in patients with SCD compared to healthy controls, and that SCIs are related to low CVR.

The objective of this study was to investigate regional CVR measurements in SCD and to investigate the association between CVR and the presence and volume of existing ischemic lesions in SCD. In the current study, we used arterial spin labelling (ASL), a non-contrast perfusion MRI method, to assess whole brain CBF prior to and following 


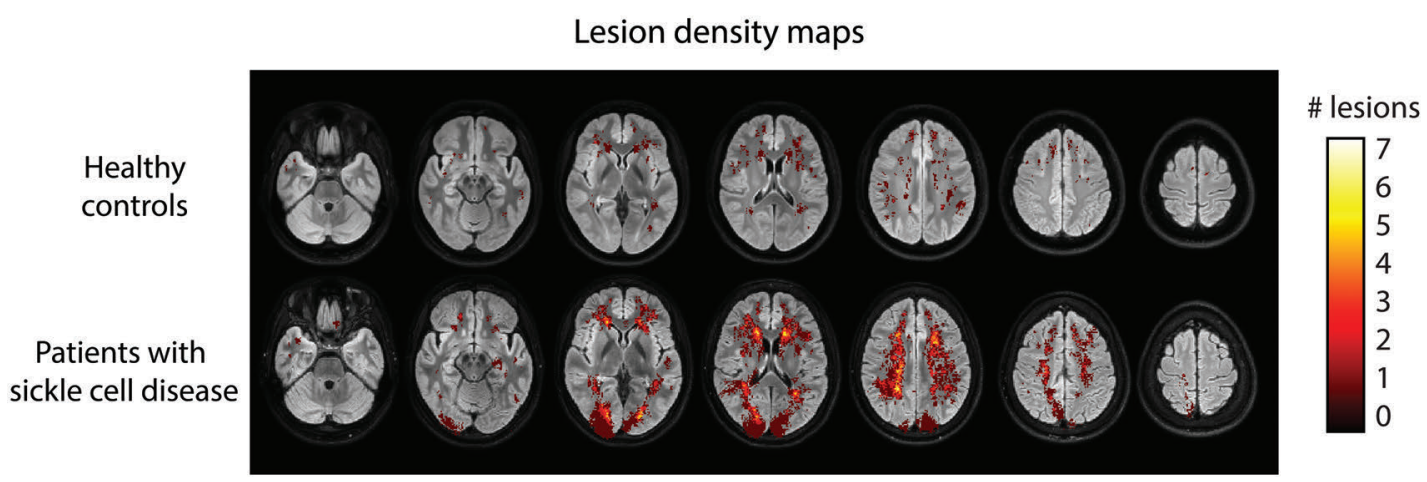

Figure 1. Lesion maps in adult patients with sickle cell disease and healthy controls. Lesions are scaled to local count of participants with a lesion. The local maximum of 2 lesions were detected in the healthy controls (upper row) and 7 in the SCD cohort (bottom row). Deep white matter, periventricular and border zone regions exhibited the highest probability of lesions. Also note the large posterior infarct in one patient in the bottom row.

cerebral vasodilation with acetazolamide. We compared hemodynamic MRI parameters between adult SCD patients in steady-state and without a history of stroke, with healthy controls.

\section{Methods}

\section{Participants}

The local Institutional Review Board at the Academic Medical Center, the Netherlands, approved this study, which was carried out in accordance with the Declaration of Helsinki. Adult patients were recruited by their SCD specialist hematologist from the outpatient clinic, and race-and age-matched controls consisted of their healthy non-SCD friends and family. Inclusion criteria were: informed consent, SCD (HbSS/HbS $\beta^{0}$-thalassemia), age (>18), and steady state (absence of an acute SCD-related event 1 month prior to participation). Exclusion criteria were: contraindications to acetazolamide or MRI, clinical history of overt infarct/hemorrhagic stroke, brain tumor, brain surgery, or serious neurologic event. Participants were asked to refrain from consuming alcohol and caffeinated drinks on the day of the examination.

\section{Order of procedures}

Participants first underwent a blood pressure measurement and blood draw. Subsequently, an intravenous catheter was placed at the site of cannulation for acetazolamide administration during the MRI scan. Blood pressure was measured before and after the $\mathrm{MRI}$ and heart rate was monitored continuously during the MRI. Subjects were asked about side-effects afterwards (Online Supplementary Table S1).

\section{Biological parameters}

Blood samples were drawn from an antecubital vein directly prior to MRI and assessed using standard laboratory procedures. Genotype was confirmed by high-performance liquid chromatography (HPLC) and DNA analysis. Missing lab data were dealt with by last steady-state observation carried forward. Markers indicating hemolysis were defined as serum levels of bilirubin, reticulocyte count and lactate dehydrogenase (LDH) ${ }^{24}$ In addition, hemoglobin concentration, $\mathrm{MCV}, \mathrm{HbF} \%, \mathrm{HbS} \%$, leukocyte count, platelet count, ASAT, ALAT, creatinine and CRP were determined.

\section{MR imaging}

We performed 3T MRI (Philips Ingenia) with a 32-channel receive head coil in all participants. For CBF, a pseudo-continuous arterial spin labelling (pCASL) sequence was used with a 2D gradient echo FFE single shot echo-planar imaging (EPI) readout with a TR/TE of 4400/14 ms, FOV $240 \times 240 \mathrm{~mm}$, voxel size $3 \times$ $3 \times 7 \mathrm{~mm}$, post-label delay $1800 \mathrm{~ms}$, label duration $1800 \mathrm{~ms}, 19$ axial slices, flip angle $90^{\circ}$, SPIR fat suppression, 140 label-control pairs, background suppression, and a total scan duration of 20 min. In addition, we acquired 3D time-of-flight magnetic resonance angiography and a 3D fluid-attenuated inversion recovery (FLAIR) sequence for lesion assessment. After 5 min of pCASL, participants received $16 \mathrm{mg} / \mathrm{kg}$ acetazolamide (Diamox®, Mercury Pharmaceuticals Ltd., London, UK) with a maximum of $1400 \mathrm{mg}$. Acetazolamide was dissolved in $20 \mathrm{~mL}$ saline $(\mathrm{NaCl}$ $0.9 \%$ ) and injected intravenously at a flow rate of $0.1 \mathrm{~mL} / \mathrm{s}$, and flushed with $10 \mathrm{~mL}$ saline. Voxel-wise CVR was calculated by: CVR $(\%)=(\triangle \mathrm{CBF}) / \mathrm{CBF}-\mathrm{PRE} \times 100 \%$, where $\Delta \mathrm{CBF}$ represents the average of the first 5 min (CBF-PRE) of the pCASL CBF timeseries subtracted from the average of the final $5 \mathrm{~min}$. We looked at gray matter (GM) and white matter (WM) CBF and CVR by applying the subject-specific anatomic masks to each subject's CBF map. CBF quantification was customized to improve the accuracy of $\mathrm{CBF}$ by using a dual compartment flow model incorporating T1 of blood, measured directly in each subject in the sagittal sinus. ${ }^{25}$ We also incorporated a labelling efficiency correction based on velocity measured with phase-contrast MRI, and a correction for the arterial transit time, measured with a separate multiple inversion time sequence. The 3T MRI protocol and image analysis is described in more detail in the Online Supplementary Methods.

\section{Lesions}

FLAIR images were manually segmented and validated by a neuroradiologist (CBM $>20$ years of experience), blinded to the medical status of the patient. We quantified voxel-wise prevalence, subject-wise prevalence, total volume, and total number of lesions. Lesions were defined as multiple $(>1)$ signal hyperintensities $\geq 5 \mathrm{~mm}$ in diameter.

These lower limits were chosen to maintain external validity with previous studies in adults with SCD. ${ }^{26-28}$ Lesion diameter was defined as the maximum length along the major axis of a lesion in 3D. Since the contribution of different types of lesions to specific impairments is not known, we also included the following lesions in the total lesion volume calculation: lacunar lesions, defined as round or ovoid subcortical fluid-filled cavities, and cortical infarcts, defined as (fluid-filled) regions of 

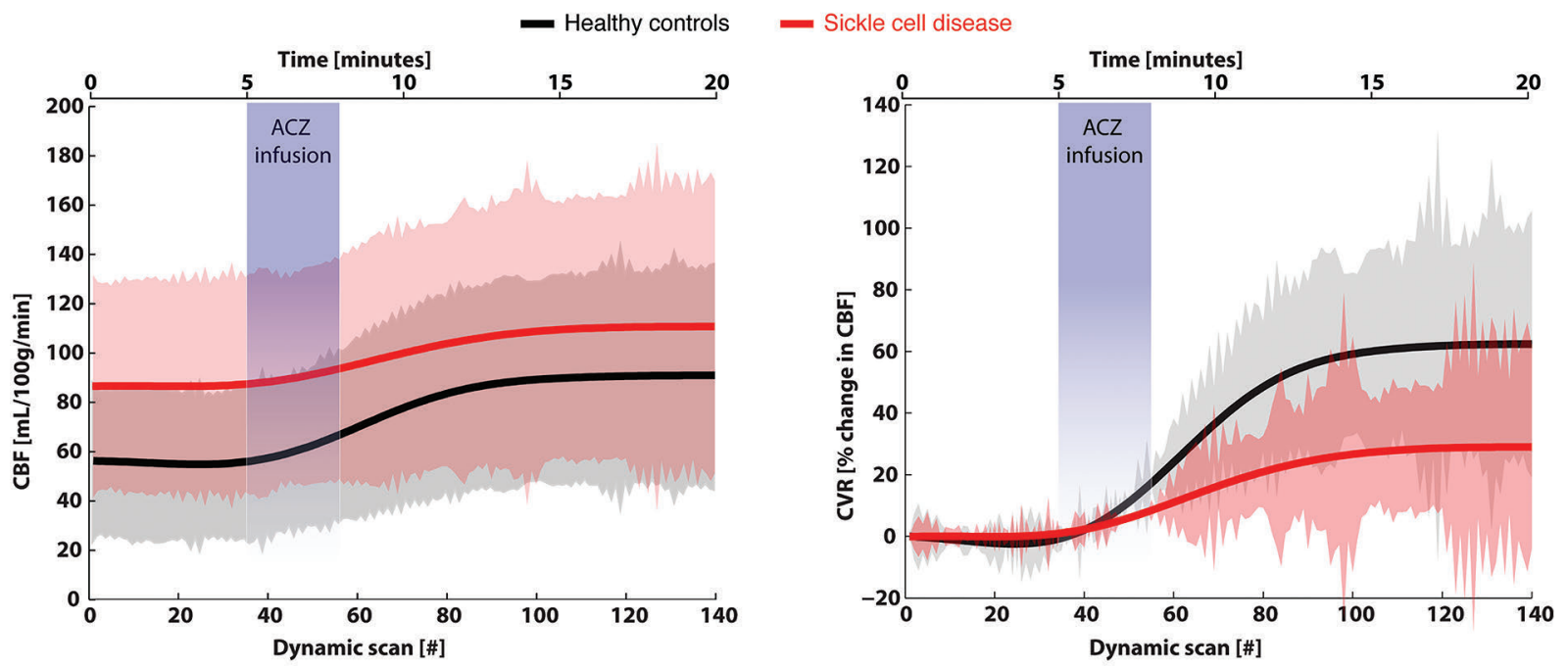

Figure 2. Dynamic gray matter CBF time-course in response to acetazolamide. Acetazolamide administration elicited a robust response in healthy controls (black solid line with grey standard deviations) and patients with sickle cell disease (SCD)(red solid line with pink standard deviations). Absolute CBF changes in the left plot indicate a higher baseline, smaller absolute increase, and slower time to rise in patients with SCD compared to healthy controls. The relative CBF in the right plot show reduced CVR in patients with SCD. Both the CBF and CVR stabilized 10-15 minutes after injection of acetazolamide.

hyperintense necrotic tissue of variable size and shape located in the cortical tissue. A lesion density map was generated by image registration (described in the Online Supplementary Methods) and lesion contours were overlaid on the CVR images to visualize co-localization.

\section{Statistics}

Statistical significance was assessed in R 3.4.3 (R Core Team (2017) R Foundation for Statistical Computing, Vienna, Austria) using appropriate tests (parametric for normally distributed and non-parametric for significantly non-normal distributed variables based on a Shapiro-Wilk test) to compare medians, means, or proportions between groups. Scatterplots of correlation analyses show both controls and patients, but exploratory correlation coefficients were computed in the patient group only, using Spearman's rho $(\rho)$. $P$-values were adjusted for multiple comparisons using the Benjamini-Hochberg method. Variables that were statistically significant $(P<0.05)$ in univariate analysis, were entered as predictor variables in multivariate analysis using the standard enter method with CVR or lesions as an outcome variable. Lesion volume was used in linear regression, while lesion presence or absence was used in binary logistic regression with CVR as a predictor variable.

\section{Results}

\section{Demographic and clinical characteristics}

Thirty-six patients and 11 healthy controls were included in the study (Table 1). Patient and control populations were well matched for age, sex, and ethnicity. HPLC and DNA analysis confirmed that $32(89 \%)$ patients had the $\mathrm{HbSS}$ genotype and $4(11 \%)$ had $\mathrm{HbS} \beta^{\circ}$ thalassemia. In the healthy control group, $2(18 \%)$ were sickle cell gene carriers (HbAS). Thirteen (37\%) patients with SCD were using hydroxyurea and $3(9 \%)$ were receiving regular (every 3-5 weeks) blood transfusions. For those on transfusions, patients were studied 3-28 days since their last transfusion. Regular blood transfusions were given to one patient for prevention of stroke upon detection of high TCD values when they were still in pediatric care, and the other patients were on transfusions for prevention of frequent hydroxycarbamide refractory vaso-occlusive crises/acute chest syndrome. Patients with SCD had lower blood pressure and body weight compared to healthy controls as well as expected differences in hematologic measurements (Table 1).

\section{Anatomic neuroimaging findings}

MRA data were of high quality, except for two patients' scans showing small motion artefacts, which precluded assessment. Incidental findings included the following: 1 patient had bilateral MCA occlusions with moyamoya syndrome and corresponding collaterals with diffuse white matter hyperintensity, 2 patients had a total of three aneurysms (smaller than $3 \mathrm{~mm}$ in diameter with a wide base), 2 patients had infundibula at the origin of the ophthalmic artery, 3 patients had tortuous vessels, and no arteriovenous malformations were found.

The prevalence of white matter, cortical, periventricular and lacunar lesions in SCD patients was 29/36 (81\%) and in healthy controls it was $5 / 11$ (45\%), $P=0.02$ (Figure 1). Two patients had cortical infarct (occipital and frontal lobes) and 5 patients had lacunar (fluid-filled cavity) infarcts. We found no lacunar or cortical infarcts in the healthy controls. Periventricular hyperintensity was observed in both groups. Patients with SCD had a similar number of lesions as compared to healthy volunteers (median [interquartile range] of 6 [19) lesions per patient versus 5 [8.5] lesions per healthy control) but significantly larger lesions with a median lesion volume of 0.34 [1.56] $\mathrm{mL}$ compared to healthy controls $(0.02[0.28] \mathrm{mL}, P=0.03)$ (Table 2). The maximum number of lesions per subject in the co-registered lesion count map in patients with SCD was seven, located in the periventricular and borderzone regions (Figure 1 ). 
Table 2. Neuroimaging findings in healthy controls and patients with sickle cell disease.

\begin{tabular}{|c|c|c|c|}
\hline & Controls (n=11) & $\begin{array}{l}\text { Patients with SCD } \\
\qquad(n=36)\end{array}$ & $P^{+*}$ \\
\hline \multicolumn{4}{|l|}{ MRA $^{\mathrm{a}}$} \\
\hline Circle of Willis variant ${ }^{\mathrm{b}}$ & 1 & 6 & 0.54 \\
\hline Hypoplasia $^{c}$ & 7 & 12 & 0.07 \\
\hline Stenosis $^{d}$ & 0 & 2 (25-50\%, occlusion) & 0.42 \\
\hline Aneurysms (no. of patients) ${ }^{e}$ & 0 & $2(6 \%)$ & 0.42 \\
\hline Infundibulum & 0 & $2(6 \%)$ & 0.42 \\
\hline Moyamoya / collaterals ${ }^{g}$ & 0 & $1(3 \%)$ & 0.58 \\
\hline Tortuous/curved vessels & 0 & 3 (ACA, MCA, PCOM) & 0.32 \\
\hline \multicolumn{4}{|l|}{ FLAIR MRI } \\
\hline Lacunar infarcts, n(\%) & 0 & $5(14 \%)$ & 0.19 \\
\hline Cortical infarcts, n(\%) & 0 & $2(6 \%)$ & 0.42 \\
\hline Periventricular infarcts, n(\%) & $1(9 \%)$ & $1(3 \%)$ & \\
\hline Total lesion count & 83 & 386 & \\
\hline Lesion count per subject, median [IQR] & $1[8]$ & $5[15]$ & $0.27^{*}$ \\
\hline Lesion volume, median mL [IQR] & $0.02[0.28]$ & $0.34[1.56]$ & $0.03 *$ \\
\hline Prevalence of lesions ( $>1$ lesion, $>5 \mathrm{~mm}$ ) & $5(45 \%)$ & $29(81 \%)$ & 0.02 \\
\hline
\end{tabular}

${ }^{+}$Chi-square test. ${ }^{*}$ Wilcoxon rank sum test. ${ }^{~}$ Normal MRA: normal defined as full Circle of Willis, excludes anatomic variants and hypoplasia. ${ }^{b}$ Variant MRA: anatomic variant of the circle of Willis such as absence of a PCOM, a fetal variant PCA, non-fusion at origin of vertebro-basilar artery, early branching of a distal artery. ${ }^{\circ}$ Hypoplasia: diameter $<1$ mm

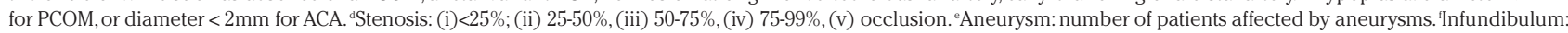
dilatational widening of the origin of a junctional artery. ${ }^{8}$ Moyamoya syndrome: bilateral occlusion of the terminal portion of ICA or proximal MCA, with abnormal vascular networks (collaterals) in the vicinity of the occlusive lesions. MRA: magnetic resonance angiogram; FLAIR MRI: fluid attenuation inversion recovery magnetic resonance imaging; SCD: sickle cell disease; ACA: anterior cerebral artery; MCA: middle cerebral artery; PCOM: posterior communicating artery; IQR: interquartile range.

\section{Dynamic cerebral blood flow response to acetazolamide}

The CBF and CVR time-series plotted in Figure 2 show that maximal dilatation was reached 10-15 minutes after acetazolamide in both patients and controls. All healthy subjects exhibited a robust response to acetazolamide without changes in blood pressure or heart rate (data not shown) measured before and after the scan. The most common side-effects after acetazolamide were dizziness, experienced by $26 \%$ of participants, headache in $13 \%$, and also paresthesia in $13 \%$. No side-effects required intervention (Online Supplementary Table S1).

\section{Cerebral hemodynamics in SCD differ from healthy controls}

The boxplots in Figure 3 show that the 36 patients with SCD had higher gray matter (GM) CBF (median [interquartile range]: 82.8 [20.1] $\mathrm{mL} / 100 \mathrm{~g} / \mathrm{min}$ ) at baseline compared to the 11 healthy controls (51.3 [4.8] $\mathrm{mL} / 100 \mathrm{~g} / \mathrm{min}, P<0.001)$. After acetazolamide, median GMCBF increased in patients to 108.3 [25.9] $\mathrm{mL} / 100 \mathrm{~g} / \mathrm{min}$, and in healthy controls to $85.5[10.8]$ $\mathrm{mL} / 100 \mathrm{~g} / \mathrm{min}, P<0.001$. Patients with SCD had $49 \%$ lower median GM CVR (34.1 [33.4] \%) compared to controls (69.5 [32.4] \%, P<0.001). Median white matter (WM) CBF was higher at baseline in SCD patients (39.6 [10.9] $\mathrm{mL} / 100 \mathrm{~g} / \mathrm{min}$ ) compared to healthy controls (26.5 [3.0] $\mathrm{mL} / 100 \mathrm{~g} / \mathrm{min}, P<0.001)$. In WM, there was a significant increase in CBF after acetazolamide in patients with SCD $(P<0.001)$, as well as in healthy controls $(P=0.002)$. Median WMCVR was $41 \%$ lower in patients with SCD compared to healthy controls (SCD: 27.1 [16.2]\%; controls: 66.1
[37.3]\%, $P<0.001)$. Figure 4 shows the the co-registered maps indicating the SCD group had higher average CBF and lower average CVR compared to healthy controls. The lowest GM CVR of $5.2 \%$ was found in the one patient who had comorbid moyamoya, and a low hemoglobin concentration of $6.4 \mathrm{~g} / \mathrm{dL}$. The small $(\mathrm{n}=3)$ group of patients receiving transfusions, and even fewer $(n=1)$ receiving transfusions but no hydroxyurea, precluded a statistical comparison of these subgroups. However, fewer days since last transfusion appeared to be associated with a trend to higher CVR, as shown in the descriptive table in Online Supplementary Table S2. Additionally, there was no difference in GM CVR between patients receiving hydroxyurea and those not receiving hydroxyurea $(P=0.89)$.

\section{Factors associated with cerebral hemodynamics}

Hematologic parameters (Table 3) were explored for their correlation with $\mathrm{CBF}$ and CVR. Resting CBF was inversely associated with age, hemoglobin concentration, erythrocyte count and $\mathrm{HbF} \%$, and positively associated with LDH and total bilirubin. After adjustment for multiple comparisons, only hemoglobin concentration remained significantly associated with resting CBF. CVR was associated with baseline CBF, hemoglobin concentration, erythrocyte count, and creatinine, and negatively associated with platelet count. After adjustment for multiple comparisons, none remained significant. In multivariate analysis, only baseline $\mathrm{CBF}$ remained significantly associated with CVR $(P=0.029)$.

\section{Cerebral hemodynamics and lesion co-localization}

We observed regional variation in the group-averaged WM CVR maps as shown in the lower panel of Figure 4. Co-localization of CVR in lesions are shown by the lesion 
Table 3. Spearman's correlation coefficients between clinical parameters and GM cerebral blood flow and GM cerebrovascular reserve in adult patients with sickle cell disease.

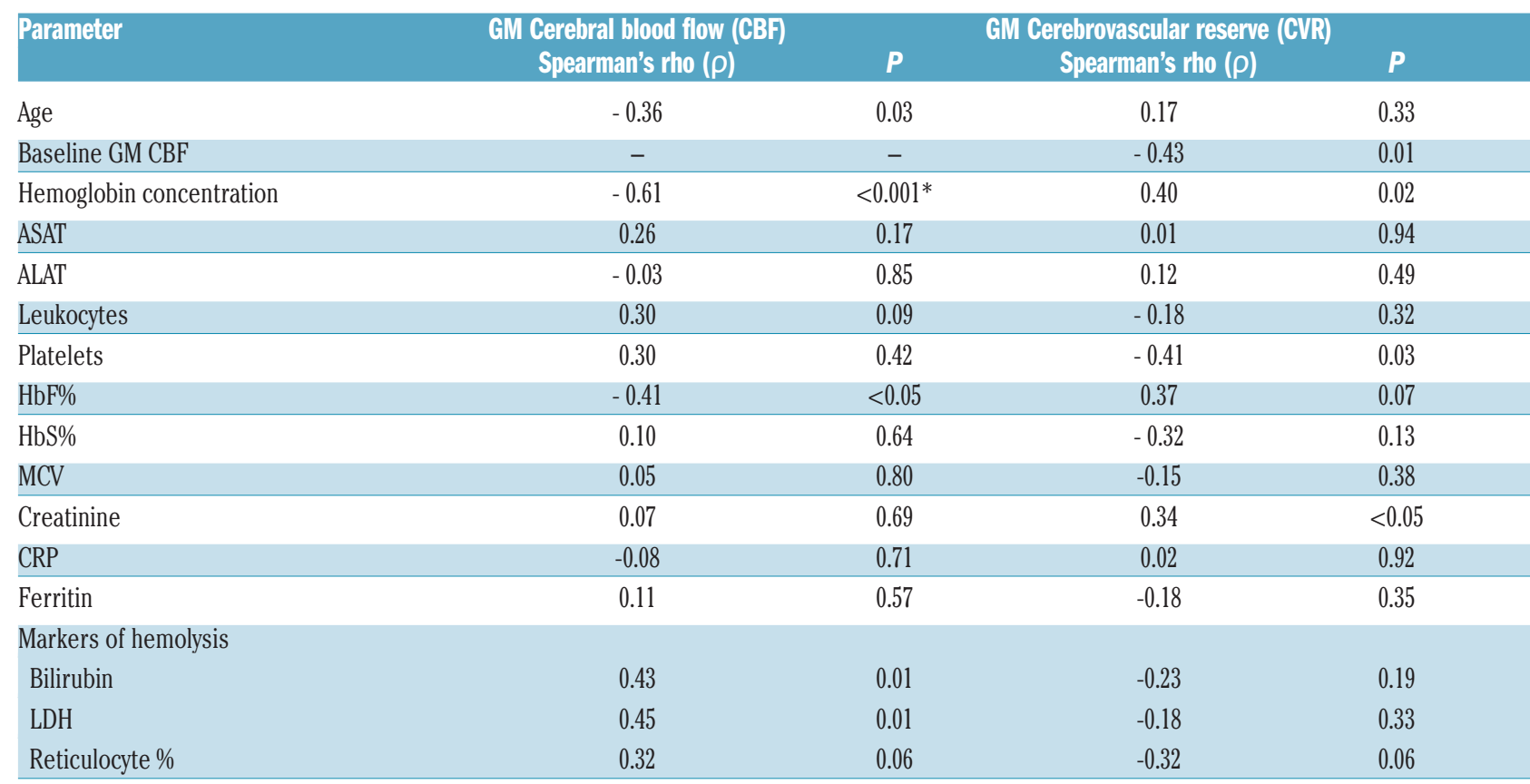

* $P$ values that remained significant after Benjamini-Hochberg procedure for multiple-comparison adjustment.GM CBF: granulocyte-macrophage cerebral blood flow; CVR: cerebrovascular reserve; ASAT: aspartate aminotransferase; ALAT: alanine aminotransferase; MCV: mean corpuscular volume; CRP: c-reactive protein; LDH: lactate dehydrogenase;

contour overlay in the bottom row of Figure 4. Binary logistic regression with lesion presence or absence as an outcome variable, showed that GM CVR was not a predictor of lesion prevalence $(P=0.28)$, and neither was WM $\operatorname{CVR}(P=0.57)$. The same was true for GM CBF $(P=0.18)$, age $(P=0.24)$, hemoglobin levels $(P=0.08)$, and the other blood markers. In linear regression analysis, CVR was not a significant predictor of lesion volume $(P=0.669)$.

\section{Discussion}

Chronic inflammation and hemolysis play a key role in the pathologic processes that can diminish the dilatory capacity of small resistance arteries in SCD. In our study, we observed a globally reduced gray matter CVR in patients with SCD without a history of stroke, compared to race-matched healthy controls. We found that CVR was particularly impaired in patients with high baseline $\mathrm{CBF}$, indicating that in these patients, cerebral vasodilation was almost maximal at rest. Indeed, the lowest CVR of $5 \%$ was observed in a patient with SCD and comorbid moyamoya syndrome, in whom the highest CBF and diffuse white matter injury was observed. Silent cerebral infarcts (SCIs) were detected in the majority of patients, but these were not related to any of the hemodynamic MRI markers. The association between CBF and elevated lactate dehydrogenase and bilirubin levels suggest that blood flow may be related to higher hemolytic rate. However, this association was not significant after adjustment for multiple comparisons so remains to be investigated in future studies.

Acetazolamide was well-tolerated by all participants and did not induce vaso-occlusive crisis in any of the patients, indicating that this test can be performed safely to assess CVR in patients with SCD. A previous study also using intravenous acetazolamide administration in children with SCD to assess CVR with SPECT ${ }^{16}$ did not report on safety of acetazolamide, so it remains unclear if the authors had the same experiences regarding side-effects. Acetazolamide has the advantage over $\mathrm{CO}_{2}$ inhalation of inducing maximal dilation without metabolic changes, which allows a true assessment of vasodilatory capacity.

We observed a plateau in the response to acetazolamide after 10-15 minutes, corresponding to maximal vasodilation. However, there was a difference in the maximal CBF between the groups, clearly showing a reduced vascular reserve capacity. This difference in CVR can be explained by chronically increased resting vessel diameter, as we have shown previously, ${ }^{21}$ which leaves these patients with little reserve for further vasodilation. Numerous resting ASL studies in children with SCD have shown that chronic anemia leads to high $\mathrm{CBF}^{20,29-32}$ and the high flow requirements in SCD could lead to a loss of autoregulatory capacity if dilatory reserve is being used for perfusion. Our dynamic CBF response supports our hypothesis that adult patients with SCD have severely reduced vasodilatory capacity. Hence, autoregulatory capacity is being used to maintain basal cerebral oxygenation, posing a risk for cerebral ischemia and infarction.

The prevalence of SCIs found in our cohort was $81 \%$, which is in line with previous reports on lesions in adults with SCD ranging from $15 \%$ to $90 \% .^{9,26-28,33}$ The large aforementioned variation arises from methodologic differences including improvements in imaging technology providing higher sensitivity, differences in age between 
A

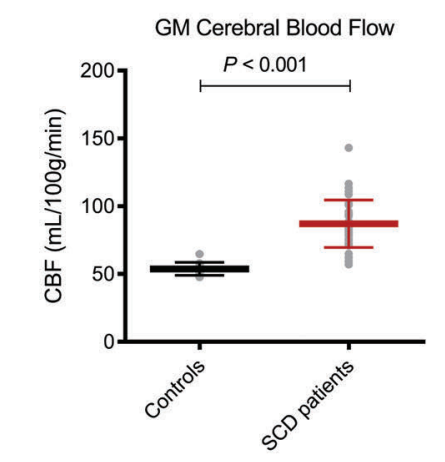

B

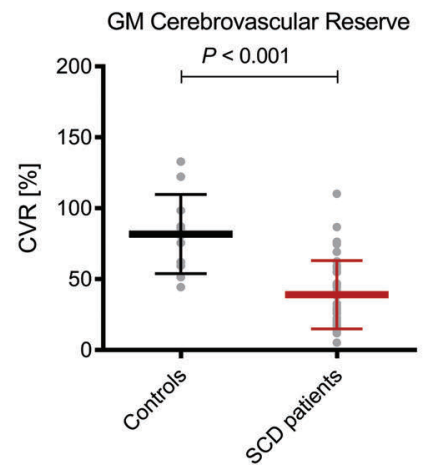

C

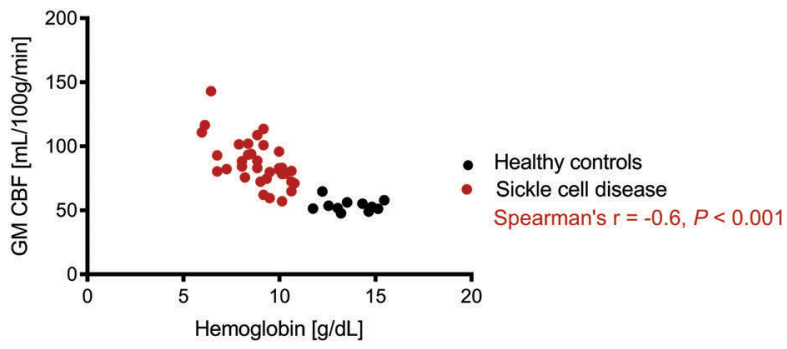

D

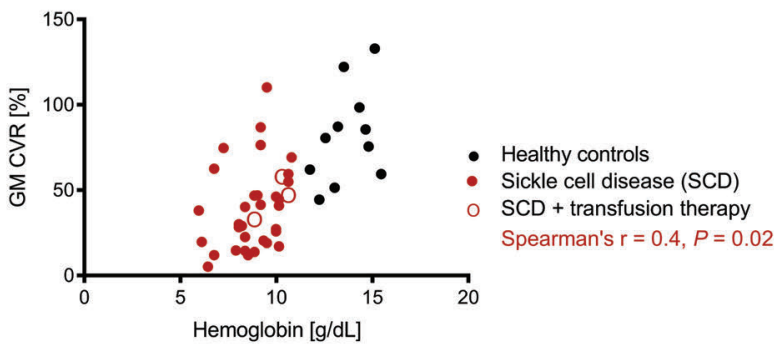

E

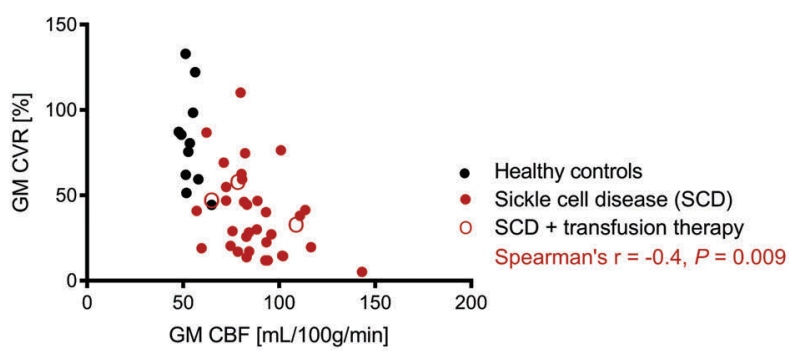

Figure 3. Differences between patients and controls, and associations among gray matter CBF, gray matter CVR, and hemoglobin. (A) The boxplot shows higher CBF in gray matter (GM) in patients with sickle cell disease (SCD) compared to healthy controls. (B) CVR in GM in patients with SCD was half of that of controls. (C) The scatterplot shows the significant association between CBF in GM and hemoglobin concentration in SCD patients. (D) The scatterplot shows that CVR in GM was significantly associated with hemoglobin levels in SCD. (E) The magnitude of the CVR in GM was significantly associated with resting CBF in GM. CBF: cerebral blood flow; CVR: cerebrovascular reserve; GM: gray matter; SCD: sickle cell disease.

patient cohorts, ${ }^{28}$ the size and number of the lesions, ${ }^{34}$ as well as differentiation between silent cerebral infarcts and lacunar infarcts. ${ }^{3,26}$ This sensitivity to technology was previously demonstrated in a study by our group performed in adult SCD patients, ${ }^{28}$ in which a 7T imaging field strength with $0.8 \mathrm{~mm}$ isotropic resolution was compared to $3 \mathrm{~T}$ with $1 \mathrm{~mm}$ isotropic resolution, and found a lesion prevalence of $50 \%$ at $3 \mathrm{~T}$ and $90 \%$ at $7 \mathrm{~T}$ field strength, respectively. The relatively high prevalence of lesions $(45 \%)$ identified in the control group seems consistent with having higher detection sensitivity when using improved technology. ${ }^{28}$ Hence, a consensus on lesion definition and measurement that is harmonized across technologies and sites is needed in order to compare studies from several cohorts in future studies.

As demonstrated in the lesion density map in our study, SCI prone areas were primarily located in the deep white matter, watershed and borderzone regions, ${ }^{35}$ where perfusion is known to be lower, and ischemic risk thought to be higher in children with SCD, ${ }^{36}$ than in the cerebral cortex. While the pathogenesis of SCIs in SCD is still unclear, some evidence does show that the severity of anemia plays a role in SCIs in children with SCD. ${ }^{37}$ Even though insufficient cerebral oxygen delivery and the associated ischemic risk is probably due to chronic anemia, recent work demonstrated that additional acute moments of critical hypoperfusion may lead to lesions rather than chronic anemia by itself. ${ }^{7,22}$ Limited cerebrovascular reserve may be the underlying condition that places patients at increased vulnerability for inadequate oxygen delivery and extraction during acute anemia or superimposed hypoxia. Hence, low CVR itself may not be a sufficient condition to initiate lesion formation, but an additional crucial 'second hit', such as acute anemic events or superimposed hypoxia, is probably necessary. We hypothesize that interventions that improve oxygen delivery such as blood transfusion, hydroxyurea or new disease-modifying drugs that reduce hemolysis may improve CVR and thereby reduce risk for cerebral ischemia and infarction. Whether reduced CVR is an independent risk factor for SCIs remains to be demonstrated in a prospective trial.

The limitations of this study include potential selection bias of patients with no history of stroke. This may have induced a bias towards less severe patients and thereby also less severe white matter injury burden. Indeed, lesions were mostly small punctate lesions, with a total median lesion volume of $0.34 \mathrm{~mL}$, which is low compared to total brain volume. However, since these lesions were present in the majority of patients, our cohort is likely to be a reasonable representation of the adult SCD population without overt stroke. We included 36 patients with SCD under the premise that we would have enough power to detect differences in CVR between patients and healthy controls based on an a priori sample size calculation. However, given that the correlation between lesion volume and CVR has not been studied previously in SCD, 


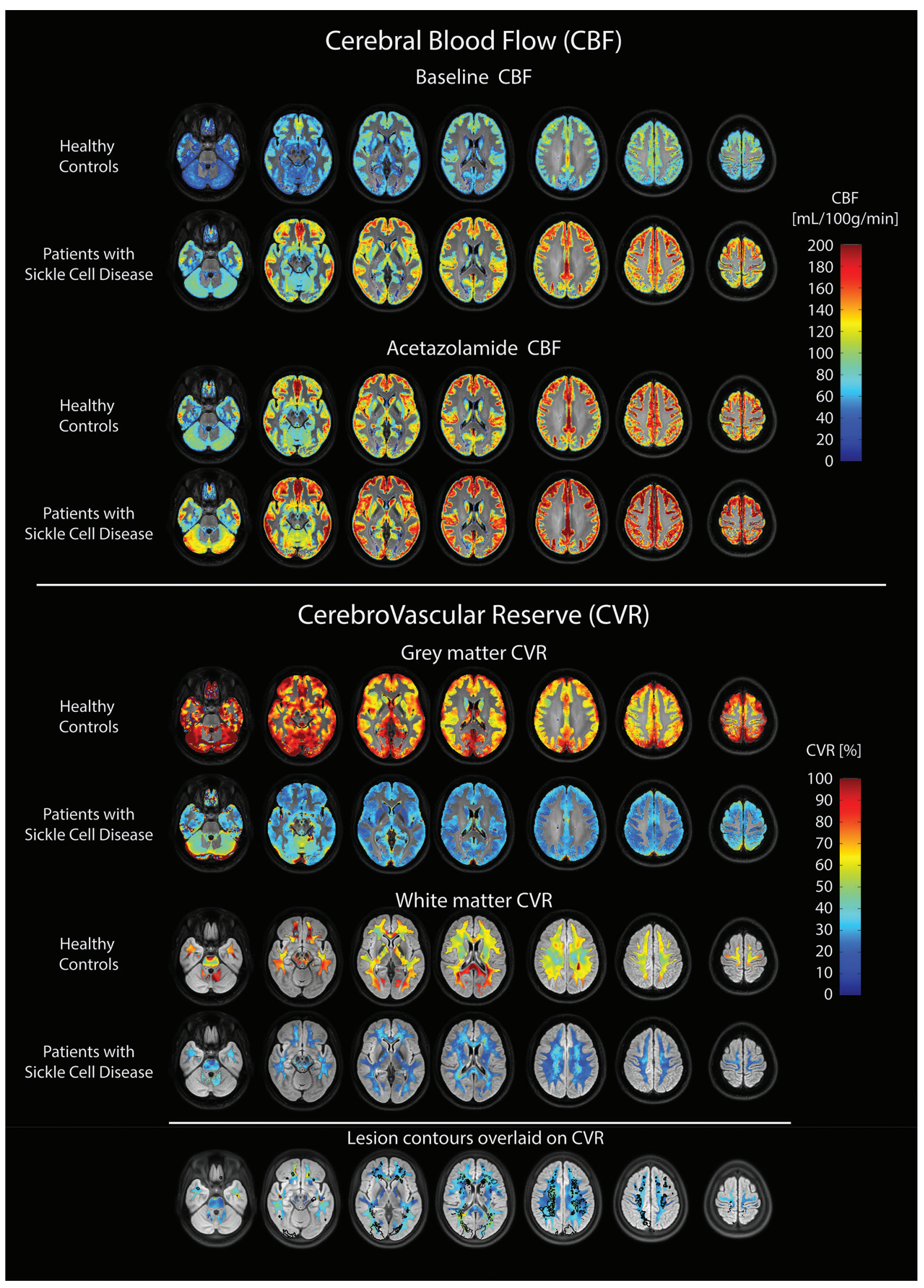

Figure 4. Axial slices of cohort averaged registered maps of hemodynamic MRI parameters. Upper panel shows CBF at baseline and post acetazolamide in GM for healthy controls and patients with sickle cell disease (SCD), and indicates clearly that CBF is elevated in SCD patients in all brain regions. Middle panel shows cohortaveraged co-registered CVR maps in GM and WM and illustrates that CVR is lower in patients with SCD compared to healthy controls in both grey and white matter regions of interest. CVR was not uniformly distributed and appeared to be higher in posterior compared to anterior regions in healthy controls and appear to be higher in watershed and periventricular regions in patients with SCD, with lowest CVR appearing in the deep white matter interface with grey matter. Bottom panel shows the lesion contours overlaid on white matter CVR in patients with SCD. CBF: cerebral blood flow; CVR: cerebrovascular reserve; GM: gray matter. 
the lack of knowledge of expected values precluded a comprehensive sample size calculation so we were possibly underpowered to detect the hypothesized negative correlation between CVR and lesion volume. Another limitation is the cross-sectional design of our study. If CVR is an early indication of hemodynamic compromise in a certain brain region, then ischemic injury may not occur until oxygen delivery is repeatedly interrupted, which may explain the fact that no association between CVR and SCI was found. Or their treatment has improved their CVR and precluded detection of the association between CVR and SCIs that had formed prior to effective therapy. Interestingly, in a previous study in non-SCD patients severely affected with white matter lesions, a link between low CVR and progression of cerebral lesions was found one year later. ${ }^{13}$ Additionally, our study may have lacked sensitivity in white matter CVR values. The reason for this is that with ASL, CBF signal in the deep white matter is often below the noise level, which makes small changes in CBF after a CVR challenge even more difficult to detect. Hence, CVR values become less reliable further away from gray matter. Improving CBF signal in white matter can be achieved by acquiring ASL for a longer duration. Improvements in ASL technology and scan acceleration will hopefully make this available for clinical research soon.

In conclusion, using ASL MRI in combination with hemodynamic provocation by acetazolamide, we demon- strated that CVR is globally reduced in adult sickle cell patients without a history of stroke. Even in steady state and at rest, patients with SCD utilize half of the cerebral vasodilatory reserve in comparison to control participants to compensate for anemia. Complete depletion of CVR can occur in the presence of additional strain on the vasculature such as in moyamoya syndrome, leading to extreme vulnerability to hypoxia and ischemic events. It remains to be seen whether increasing hemoglobin levels by transfusion, hydroxyurea or new disease-modifying drugs can relieve some of the restrictions imposed on the brain by anemia and whether they also reduce cerebral infarction.

\section{Acknowledgments}

The authors would like to thank all the participants involved in this study, Magdalena J Sokolska and David L Thomas for their expertise and contribution to the simulations on labelling efficiency for the pCASL sequence implementation, Moss Y Zhao and Michael Chappell for their contribution to the arterial transit time calculations, Sandra van den Berg and Raschel van Luijk for their excellent clinical and MR support, Erfan Nur and Charlotte van Tuijn for their help with recruitment and clinical expertise and Jan Petr for assistance with the ASL data analysis.

\section{Funding}

We wish to thank the Dutch fund "Fonds Nuts Ohra" for funding this research (grant no. 1303-055).

\section{References}

1. Rees DC, Williams TN, Gladwin MT. Sickle-cell disease. Lancet. 2010;376(9757): 2018-2031.

2. Kato GJ, Gladwin MT, Steinberg $M H$. Deconstructing sickle cell disease: Reappraisal of the role of hemolysis in the development of clinical subphenotypes. Blood Rev. 2007;21(1):37-47

3. DeBaun MR, Armstrong FD, McKinstry RC, et al. Silent cerebral infarcts: a review on a prevalent and progressive cause of neurologic injury in sickle cell anemia. Blood. 2012;119(20):4587-4597.

4. Prengler M, Pavlakis SG, Prohovnik I, Adams RJ. Sickle cell disease: the neurological complications. Ann Neurol. 2002; 51(5):543-52.

5. van der Land V, Hijmans CT, de Ruiter M, et al. Volume of white matter hyperintensities is an independent predictor of intelligence quotient and processing speed in children with sickle cell disease. $\mathrm{Br} J$ Haematol. 2015;168(4):553-556.

6. Miller ST, Macklin EA, Pegelow CH, et al. Silent infarction as a risk factor for overt stroke in children with sickle cell anemia: A report from the Cooperative Study of Sickle Cell Disease. J Pediatr. 2001; 139(3):385-390.

7. Bernaudin F, Verlhac S, Arnaud C, et al. Chronic and acute anemia and extracranial internal carotid stenosis are risk factors for silent cerebral infarcts in sickle cell anemia. Blood. 2015;125(10):1653-1661.

8. DeBaun, MR, \& Kirkham, FJ. Central nervous system complications and management in sickle cell disease. Blood, 2016;127(7):829-838.
9. Kassim AA, Pruthi S, Day M, et al. Silent cerebral infarcts and cerebral aneurysms are prevalent in adults with sickle cell anemia - Letter to the Editor. Blood. 2016; 127(16):2038-2041.

10. DeBaun MR, Gordon M, McKinstry RC, et al. Controlled trial of transfusions for silent cerebral infarcts in sickle cell anemia. $\mathrm{N}$ Engl J Med. 2014;371(8):699-710.

11. Juttukonda MR, Donahue MJ. Neuroimaging of vascular reserve in patients with cerebrovascular diseases. Neuroimage. 2017 Oct 12. [Epub ahead of print]

12. Settakis G, Molnár C, Kerényi L, et al. Acetazolamide as a vasodilatory stimulus in cerebrovascular diseases and in conditions affecting the cerebral vasculature. Eur J Neurol. 2003;10(6):609-620.

13. Sam K, Crawley AP, Conklin J, et al. Development of white matter hyperintensity is preceded by reduced cerebrovascular reactivity. Ann Neurol. 2016;80(2):277285.

14. Ogasawara K, Ogawa A, Terasaki K, et al. Use of cerebrovascular reactivity in patients with symptomatic major cerebral artery occlusion to predict 5-year outcome: comparison of xenon-133 and iodine-123IMP single-photon emission computed tomography. J Cereb Blood Flow Metab. 2002;22(9):1142-1148.

15. Silvestrini M, Vernieri F, Pasqualetti $P$, et al. Impaired cerebral vasoreactivity and risk of stroke in patients with asymptomatic carotid artery stenosis. JAMA. 2000; 283(16):2122-2127

16. Kedar A, Drane WE, Shaeffer D, Nicole M, Adams C. Measurement of cerebrovascular flow reserve in pediatric patients with sick- le cell disease. Pediatr Blood Cancer. 2006; 46(2):234-238.

17. Nur E, Kim Y-S, Truijen J, et al. Cerebrovascular reserve capacity is impaired in patients with sickle cell disease. Blood. 2009;114(16):3473-3478.

18. Prohovnik I, Hurlet-Jensen A, Adams R, De Vivo D, Pavlakis SG. Hemodynamic etiology of elevated flow velocity and stroke in sickle-cell disease. J Cereb Blood Flow Metab. 2009;29(4):803-810.

19. Leung J, Duffin J, Fisher JA, Kassner A MRI-based cerebrovascular reactivity using transfer function analysis reveals temporal group differences between patients with sickle cell disease and healthy controls. NeuroImage Clin. 2016;12:624-630.

20. Kosinski PD, Croal PL, Leung J, et al. The severity of anaemia depletes cerebrovascular dilatory reserve in children with sickle cell disease: a quantitative magnetic resonance imaging study. Br J Haematol. 2017;1 76(2):280-287.

21. Václavů L, Baldew ZA, Gevers S, et al Intracranial 4D flow magnetic resonance imaging reveals altered haemodynamics in sickle cell disease. Br J Haematol. 2018; 180(3):432-442

22. Dowling M, Quinn C, Plumb P, et al. Acute silent cerebral infarction occurs during acute anemic events in children with and without sickle cell disease. Blood. 2012; 120(19):3891-3897

23. Kirkham FJ, Hewes DKM, Prengler M, et al. Nocturnal hypoxaemia and central-nervous-system events in sickle-cell disease. Lancet. 2001;357(9269):1656-1659.

24. Kato GJ, Steinberg MH, Gladwin MT, et al. Intravascular hemolysis and the pathophysiology of sickle cell disease. J Clin 
Invest. 2017;127(3):750-760.

25. Václavù L, van der Land V, Heijtel DF, et al. In vivo T1 of blood measurements in children with sickle cell disease improve cerebral blood flow quantification from arterial spin-labeling MRI. Am J Neuroradiol. 2016; 37(9):1727-1732

26. Vichinsky EP, Neumayr LD, Gold JI, et al. Neuropsychological dysfunction and neuroimaging abnormalities in neurologically intact adults with sickle cell anemia. JAMA. 2010;303(18):1823-1831

27. Calvet D, Tuilier T, Mélé N, et al. Low fetal hemoglobin percentage is associated with silent brain lesions in adults with homozygous sickle cell disease. Blood Adv. 2017; 1(26):2503-2509.

28. van Der Land V, Zwanenburg JJM, Fijnvandraat $\mathrm{K}$, et al. Cerebral lesions on 7 Tesla MRI in patients with sickle cell anemia. Cerebrovasc. Dis. 2015;39(3-4):181-189.

29. Gevers S, Nederveen AJ, Fijnvandraat K, et al. Arterial spin labeling measurement of cerebral perfusion in children with sickle cell disease. J Magn Reson Imaging. 2012; 35(4):779-787.

30. Kawadler JM, Hales PW, Barker S, et al. Cerebral perfusion characteristics show differences in younger versus older children with sickle cell anaemia: Results from a multiple-inflow-time arterial spin labelling study. NMR Biomed. 2018;31(6):1-11.

31. Guilliams KP, Fields ME, Ragan DK, et al. Red cell exchange transfusions lower cerebral blood flow and oxygen extraction fraction in pediatric sickle cell anemia. Blood. 2017;131(9):1012-1021

32. Croal P, Leung J, Kosinski P, et al. Assessment of cerebral blood flow with magnetic resonance imaging in children with sickle cell disease: A quantitative comparison with transcranial Doppler ultrasonography. Brain Behav. 2017; 7(11): e00811.

33. Solomou E, Kraniotis P, Kourakli A, Petsas
T. Extent of silent cerebral infarcts in adult sickle-cell disease patients on magnetic resonance imaging: Is there a correlation with the clinical severity of disease? Hematol. Rep. 2013;5(1):8-12.

34. Liem RI, Liu J, Gordon $\mathrm{MO}$, et al. Reproducibility of detecting silent cerebral infarcts in pediatric sickle cell anemia. Child Neurol. 2014;29(12):1685-1691.

35. Mangla R, Kolar B, Almast J, Ekholm SE. Border zone infarcts: pathophysiologic and imaging characteristics. Radiographics. 2011;31(5):1201-1214.

36. Ford AL, Ragan DK, Fellah S, et al. Silent infarcts in sickle cell anemia occur in the borderzone region and are associated with low cerebral blood flow. Blood. 2018; 132(16):1714-1723.

37. Kwiatkowski JL, Zimmerman RA, Pollock AN, et al. Silent infarcts in young children with sickle cell disease. Br J Haematol. 2009;146(3):300-305. 